Proceedings of the International Congress of Mathematicians

Hyderabad, India, 2010

\title{
The classification of $p$-compact groups and homotopical group theory
}

\author{
Jesper Grodal *
}

\begin{abstract}
We survey some recent advances in the homotopy theory of classifying spaces, and homotopical group theory. We focus on the classification of $p$-compact groups in terms of root data over the $p$-adic integers, and discuss some of its consequences e.g. for finite loop spaces and polynomial cohomology rings.
\end{abstract}

Mathematics Subject Classification (2000). Primary: 55R35; Secondary: 55R37, 55P35, 20F55.

Keywords. Homotopical group theory, classifying space, $p$-compact group, reflection group, finite loop space, cohomology ring.

Groups are ubiquitous in real life, as symmetries of geometric objects. For many purposes in mathematics, for instance in bundle theory, it is however not the group itself but rather its classifying space, which takes center stage. The classifying space encodes the group multiplication directly in a topological space, to be studied and manipulated using the toolbox of homotopy theory. This leads to the idea of homotopical group theory, that one should try to do group theory in terms of classifying spaces.

The idea that there should be a homotopical version of group theory is an old one. The seeds were sown already in the 40s and 50s with the work of Hopf and Serre on finite $H$-spaces and loop spaces, and these objects were intensely studied in the 60s using the techniques of Hopf algebras, Steenrod operations, etc., in the hands of Browder, Thomas, and others. A bibliography containing 347 items was collected by James in 1970 [59]; see also 62] for a continuation.

In the same year, Sullivan, in his widely circulated MIT notes [95, 94, laid out a theory of $p$-completions of topological spaces, which had a profound influence on the subject. On the one hand it provided an infusion of new exotic examples, laying old hopes and conjectures to rest. On the other hand his theory of $p$-completions seemed to indicate that the dream of doing group theory on the level of classifying spaces could still be valid, if one is willing to replace real life, at least temporarily, by a $p$-adic existence. However, the tools for seriously digging into the world of $p$ complete spaces were at the time insufficient, a stumbling block being the so-called Sullivan conjecture [95, p. 179] relating fixed-points to homotopy fixed-points, at a prime $p$.

\footnotetext{
* Supported by an ESF EURYI award and the Danish National Research Foundation.
} 
The impasse ended with the solution of the Sullivan conjecture by Miller [69], and the work of Carlsson [25, reported on at this congress in 1986 [70, 26], followed by the development of "Lannes theory" 63, 64] giving effective tools for calculating homotopy fixed-points and maps between classifying spaces. This led to a spate of progress. Dwyer and Wilkerson 42 defined the notion of a $p$-compact group, a $p$ complete version of a finite loop space, and showed that these objects posses much of the structure of compact Lie groups: maximal tori, Weyl groups, etc. In parallel to this, Jackowski, McClure, and Oliver [55] combined Lannes theory with spacelevel decomposition techniques and sophisticated homological algebra calculations to get precise information about maps between classifying spaces of compact Lie groups, that used to be out of reach. These developments were described at this congress in 1998 36, 82.

The aim here is to report on some recent progress, building on the above mentioned achievements. In particular, a complete classification of $p$-compact groups has recently been obtained in collaborations involving the author [9, 8]. It states that connected $p$-compact groups are classified by their root data over the $p$-adic integers $\mathbb{Z}_{p}$ (once defined!), completely analogously to the classification of compact connected Lie groups by root data over $\mathbb{Z}$. It has in turn allowed for the solution of a number of problems and conjectures dating from the 60 s and 70 s, such as the Steenrod problem of realizing polynomial cohomology rings and the so-called maximal torus conjecture giving a completely homotopical description of compact Lie groups. By local-to-global principles the classification of $p$-compact groups furthermore provides a quite complete understanding of what finite loop spaces look like, integrally as well as rationally.

Homotopical group theory has branched out considerably over the last decade. There is now an expanding theory of homotopical versions of finite groups, the socalled $p$-local finite groups, showing signs of strong connections to deep questions in finite group theory, such as the classification of finite simple groups. There has been progress on homotopical group actions, providing in some sense a homotopical version of the "geometric representation theory" of tom Dieck 97. And there is even evidence that certain aspects of the theory might extend to Kac-Moody groups and other classes of groups. We shall only be able to provide very small appetizers to some of these last developments, but we hope that they collectively serve as an inspiration to the reader to try to take a more homotopical approach to his or her favorite class of groups.

This paper is structured as follows: Section 1 is an algebraic prelude, discussing the theory of $\mathbb{Z}_{p}$-root data - the impatient reader can skip it at first, referring back to it as needed. Section 2 gives the definition and basic properties of $p$ compact groups, states the classification theorem, and outlines its proof. It also presents various structural consequences for $p$-compact groups. Section 3 discusses applications to finite loop spaces such as an algebraic parametrization of finite loop spaces and the solution of the maximal torus conjecture. Section 4 presents the solution of the Steenrod problem of realizing polynomial cohomology rings, and finally Section 5 provides brief samples of other topics in homotopical group theory.

Notation: Throughout this paper, the word "space" will mean "topological space 
of the homotopy type of a CW-complex".

Acknowledgments: I would like to thank Kasper Andersen, Bill Dwyer, Haynes Miller, and Bob Oliver for providing helpful comments on a preliminary version of this paper. I take the opportunity to thank my coauthors on the various work reported on here, and in particular express my gratitude to Kasper Andersen for our mathematical collaboration and sparring through the years.

\section{Root data over the $p$-adic integers}

In standard Lie theory, root data classify compact connected Lie groups as well as reductive algebraic groups over algebraically closed fields. A root datum is usually packaged as a quadruple $\left(M, \Phi, M^{\vee}, \Phi^{\vee}\right)$ of roots $\Phi$ and coroots $\Phi^{\vee}$ in a $\mathbb{Z}$-lattice $M$ and its dual $M^{\vee}$, satisfying some conditions [33. For $p$-compact groups the lattices that come up are lattices over the $p$-adic integers $\mathbb{Z}_{p}$, rather than $\mathbb{Z}$, so the concept of a root datum needs to be tweaked to make sense also in this setting, and one must carry out a corresponding classification. In this section we produce a short summary of this theory, based on $79,45,6,8$. In what follows $R$ denotes a principal ideal domain of characteristic zero.

The starting point is the theory of reflection groups, surveyed e.g. in [47. A finite $R$-reflection group is a pair $(W, L)$ such that $L$ is a finitely generated free $R$-module and $W \subseteq \operatorname{Aut}_{R}(L)$ is a finite subgroup generated by reflections, i.e., non-trivial elements $\sigma$ that fix an $R$-submodule of corank one.

Reflection groups have been classified for several choices of $R$, the most wellknown cases being the classification of finite real and rational reflection groups in terms of certain Coxeter diagrams [53. Finite complex reflection groups were classified by Shephard-Todd [89] in 1954. The main (irreducible) examples in the complex case are the groups $G(m, s, n)$ of $n \times n$ monomial matrices with nonzero entries being $m$ th roots of unity and determinant an $(\mathrm{m} / \mathrm{s})$ th root of unity, where $s \mid m$; in addition to this there are 34 exceptional cases usually named $G_{4}$ to $G_{37}$. From the classification over $\mathbb{C}$ one can obtain a classification over $\mathbb{Q}_{p}$ as the sublist whose character field $\mathbb{Q}(\chi)$ is embeddable in $\mathbb{Q}_{p}$. This was examined by Clark-Ewing [31, and we list their result in Table 1, using the original notation.

The ring $\mathbb{Q}_{p}[L]^{W}$ of $W$-invariant polynomial functions on $L$ is polynomial if and only if $W$ is a reflection group, by the Shephard-Todd-Chevalley theorem 11, Thm. 7.2.1]; the column degrees in Table 1 lists the degrees of the generators, and the number of degrees equals the rank of $(W, L)$. For many $W$, none of the primes listed in the last column divide $|W|$; in fact this can only happen in the infinite families, and in the sporadic examples $12,24,28,29,31$, and $34-37$. It is a good exercise to look for the Weyl groups of the various simple compact Lie groups in the table, where they have character field $\mathbb{Q}$. One may observe that for $p=2$ and 3 there is only one exotic reflection group (i.e., irreducible with $\mathbb{Q}(\chi) \neq \mathbb{Q}$ ), namely $G_{24}$ and $G_{12}$ respectively, whereas for $p \geq 5$ there are always infinitely many.

The classification over $\mathbb{Q}_{p}$ can be lifted to a classification over $\mathbb{Z}_{p}$, but instead of stating this now, we proceed directly to root data. 


\begin{tabular}{|c|c|c|c|c|c|}
\hline $\mathrm{W}$ & Order & Degrees & $\mathbb{Q}(\chi)$ & \multicolumn{2}{|l|}{ Primes } \\
\hline (family 1) & $(n+1) !$ & $2,3, \ldots, n+1$ & $\mathbb{Q}$ & \multicolumn{2}{|l|}{ all $p$} \\
\hline $\begin{array}{ll}G(m, s, n) & (\text { family } 2 \mathrm{a}) \\
m \geq 2, n \geq 2, m \neq s \text { if } n=2\end{array}$ & $n ! m^{n-1} \frac{m}{s}$ & $m, 2 m, \ldots,(n-1) m, n \frac{m}{s}$ & $\mathbb{Q}\left(\zeta_{m}\right)$ & \multicolumn{2}{|c|}{$\begin{array}{l}p \equiv 1 \quad(m) \\
\text { all } p \text { for } m=2\end{array}$} \\
\hline $\begin{array}{l}\left.D_{2 m}=G(m, m, 2) \text { (family } 2 \mathrm{~b}\right) \\
m \geq 3\end{array}$ & $2 m$ & $2, m$ & $\mathbb{Q}\left(\zeta_{m}+\zeta_{m}^{-1}\right)$ & \multicolumn{2}{|c|}{$\begin{array}{l}p \equiv \pm 1 \quad(m) ; \\
\text { all } p \text { for } m=3,4,6\end{array}$} \\
\hline $\begin{array}{l}C_{m}=G(m, 1,1) \quad(\text { family } 3) \\
m \geq 2\end{array}$ & $m$ & $m$ & $\mathbb{Q}\left(\zeta_{m}\right)$ & \multicolumn{2}{|c|}{$\begin{array}{l}p \equiv 1 \quad(m) \\
\text { all } p \text { for } m=2\end{array}$} \\
\hline$G_{4}$ & 24 & 4,6 & $\mathbb{Q}\left(\zeta_{3}\right)$ & $p \equiv 1$ & (3) \\
\hline$G_{5}$ & 72 & 6,12 & $\mathbb{Q}\left(\zeta_{3}\right)$ & $p \equiv 1$ & (3) \\
\hline$G_{6}$ & 48 & 4,12 & $\mathbb{Q}\left(\zeta_{12}\right)$ & $p \equiv 1$ & (12) \\
\hline$G_{7}^{0}$ & 144 & 12,12 & $\mathbb{Q}\left(\zeta_{12}\right)$ & $p \equiv 1$ & (12) \\
\hline$G_{8}$ & 96 & 8,12 & $\mathbb{Q}\left(\zeta_{4}\right)$ & $p \equiv 1$ & (4) \\
\hline$G_{9}^{\circ}$ & 192 & 8,24 & $\mathbb{Q}\left(\zeta_{8}\right)$ & $p \equiv 1$ & (8) \\
\hline$G_{10}$ & 288 & 12,24 & $\mathbb{Q}\left(\zeta_{12}\right)$ & $p \equiv 1$ & (12) \\
\hline$G_{11}$ & 576 & 24,24 & $\mathbb{Q}\left(\zeta_{24}\right)$ & $p \equiv 1$ & (24) \\
\hline$G_{12}$ & 48 & 6,8 & $\mathbb{Q}(\sqrt{-2})$ & $p \equiv 1,3$ & $(8)$ \\
\hline$G_{13}$ & 96 & 8,12 & $\mathbb{Q}\left(\zeta_{8}\right)$ & $p \equiv 1$ & (8) \\
\hline$G_{14}$ & 144 & 6,24 & $\mathbb{Q}\left(\zeta_{3}, \sqrt{-2}\right)$ & $p \equiv 1,19$ & (24) \\
\hline$G_{15}^{14}$ & 288 & 12,24 & $\mathbb{Q}\left(\zeta_{24}\right)$ & $p \equiv 1$ & $(24)$ \\
\hline$G_{16}$ & 600 & 20,30 & $\mathbb{Q}\left(\zeta_{5}\right)$ & $p \equiv 1$ & $(5)$ \\
\hline$G_{17}$ & 1200 & 20,60 & $\mathbb{Q}\left(\zeta_{20}\right)$ & $p \equiv 1$ & (20) \\
\hline$G_{18}$ & 1800 & 30,60 & $\mathbb{Q}\left(\zeta_{15}\right)$ & $p \equiv 1$ & (15) \\
\hline$G_{19}$ & 3600 & 60,60 & $\mathbb{Q}\left(\zeta_{60}\right)$ & $p \equiv 1$ & $(60)$ \\
\hline$G_{20}$ & 360 & 12,30 & $\mathbb{Q}\left(\zeta_{3}, \sqrt{5}\right)$ & $p \equiv 1,4$ & (15) \\
\hline$G_{21}$ & 720 & 12,60 & $\mathbb{Q}\left(\zeta_{12}, \sqrt{5}\right)$ & $p \equiv 1,49$ & $(60)$ \\
\hline$G_{22}$ & 240 & 12,20 & $\mathbb{Q}\left(\zeta_{4}, \sqrt{5}\right)$ & $p \equiv 1,9$ & (20) \\
\hline$G_{23}$ & 120 & $2,6,10$ & $\mathbb{Q}(\sqrt{5})$ & $p \equiv 1,4$ & (5) \\
\hline$G_{24}$ & 336 & $4,6,14$ & $\mathbb{Q}(\sqrt{-7})$ & $p \equiv 1,2,4$ & (7) \\
\hline$G_{25}^{24}$ & 648 & $6,9,12$ & $\mathbb{Q}\left(\zeta_{3}\right)$ & $p \equiv 1$ & (3) \\
\hline$G_{26}$ & 1296 & $6,12,18$ & $\mathbb{Q}\left(\zeta_{3}\right)$ & $p \equiv 1$ & (3) \\
\hline$G_{27}$ & 2160 & $6,12,30$ & $\mathbb{Q}\left(\zeta_{3}, \sqrt{5}\right)$ & $p \equiv 1,4$ & (15) \\
\hline$G_{28}$ & 1152 & $2,6,8,12$ & $\mathbb{Q}$ & all $p$ & \\
\hline$G_{29}$ & 7680 & $4,8,12,20$ & $\mathbb{Q}\left(\zeta_{4}\right)$ & $p \equiv 1$ & (4) \\
\hline$G_{30}$ & 14400 & $2,12,20,30$ & $\mathbb{Q}(\sqrt{5})$ & $p \equiv 1,4$ & (5) \\
\hline$G_{31}$ & $64 \cdot 6 !$ & $8,12,20,24$ & $\mathbb{Q}\left(\zeta_{4}\right)$ & $p \equiv 1$ & (4) \\
\hline$G_{32}$ & $216 \cdot 6 !$ & $12,18,24,30$ & $\mathbb{Q}\left(\zeta_{3}\right)$ & $p \equiv 1$ & (3) \\
\hline$G_{33}$ & $72 \cdot 6 !$ & $4,6,10,12,18$ & $\mathbb{Q}\left(\zeta_{3}\right)$ & $p \equiv 1$ & (3) \\
\hline$G_{34}$ & $108 \cdot 9 !$ & $6,12,18,24,30,42$ & $\mathbb{Q}\left(\zeta_{3}\right)$ & $p \equiv 1$ & (3) \\
\hline$G_{35}$ & $72 \cdot 6 !$ & $2,5,6,8,9,12$ & $\mathbb{Q}$ & all $p$ & \\
\hline$G_{36}$ & $8 \cdot 9 !$ & $2,6,8,10,12,14,18$ & $\mathbb{Q}$ & all $p$ & \\
\hline$G_{37}$ & $192 \cdot 10 !$ & $2,8,12,14,18,20,24,30$ & $\mathbb{Q}$ & all $p$ & \\
\hline
\end{tabular}

Table 1. The irreducible $\mathbb{Q}_{p}$-reflection groups

Definition 1.1. An $R$-root datum $\mathbf{D}$ is a triple $\left(W, L,\left\{R b_{\sigma}\right\}\right)$, where $(W, L)$ is a finite $R$-reflection group, and $\left\{R b_{\sigma}\right\}$ is a collection of rank one submodules of $L$, indexed by the set of reflections $\sigma$ in $W$, and satisfying that $\operatorname{im}(1-\sigma) \subseteq R b_{\sigma}$ (coroot condition) and $w\left(R b_{\sigma}\right)=R b_{w \sigma w^{-1}}$ for all $w \in W$ (conjugation invariance).

An isomorphism of $R$-root data $\varphi: \mathbf{D} \rightarrow \mathbf{D}^{\prime}$ is defined to be an isomorphism $\varphi: L \rightarrow L^{\prime}$ such that $\varphi W \varphi^{-1}=W^{\prime}$ as subgroups of $\operatorname{Aut}\left(L^{\prime}\right)$ and $\varphi\left(R b_{\sigma}\right)=$ $R b_{\varphi \sigma \varphi^{-1}}^{\prime}$ for every reflection $\sigma \in W$. The element $b_{\sigma} \in L$, determined up to a unit in $R$, is called the coroot corresponding to $\sigma$. The coroot condition ensures that given $\left(\sigma, b_{\sigma}\right)$ we can define a root $\beta_{\sigma}: L \rightarrow R$ via the formula

$$
\sigma(x)=x-\beta_{\sigma}(x) b_{\sigma}
$$

The classification of $R$-root data of course depends heavily on $R$. For $R=\mathbb{Z}$ root data correspond bijectively to classically defined root data $\left(M, \Phi, M^{\vee}, \Phi^{\vee}\right)$ via the association $\left(W, L,\left\{\mathbb{Z} b_{\sigma}\right\}\right) \rightsquigarrow\left(L^{*},\left\{ \pm \beta_{\sigma}\right\}, L,\left\{ \pm b_{\sigma}\right\}\right)$. One easily checks that $R b_{\sigma} \subseteq \operatorname{ker}(N)$, where $N=1+\sigma+\ldots+\sigma^{|\sigma|-1}$ is the norm element, so giving an $R$-root datum with underlying reflection group $(W, L)$ corresponds to choosing a cyclic $R$-submodule of $H^{1}(\langle\sigma\rangle ; L)$ for each conjugacy class of reflections $\sigma$. It is hence in practice not hard to parametrize all possible $R$-root data supported by a given finite $R$-reflection group. For $R=\mathbb{Z}_{p}, p$ odd, reflections have order dividing 
$p-1$, hence prime to $p$, so here $\mathbb{Z}_{p}$-root data coincides with finite $\mathbb{Z}_{p}$-reflection groups. For $R=\mathbb{Z}$ or $\mathbb{Z}_{2}$ the difference between the two notions only occur for the root data of $\mathrm{Sp}(n)$ and $\mathrm{SO}(2 n+1)$, but due to the ubiquity of $\mathrm{SU}(2)$ and $\mathrm{SO}(3)$ this distinction turns out to be an important one. Note that since a root and a coroot $\left(\beta_{\sigma}, b_{\sigma}\right)$ determine the reflection $\sigma$ by (1.1), one could indeed have defined a root datum as a set of pairs $\left(\beta_{\sigma}, b_{\sigma}\right)$, each determined up to a unit and subject to certain conditions; see also [76].

The relationship between $\mathbb{Z}_{p}$-root data and $\mathbb{Z}$-root data is given as follows.

Theorem 1.2 (The classification of $\mathbb{Z}_{p}$-root data, splitting version).

1. Any $\mathbb{Z}_{p}$-root datum $\mathbf{D}$ can be written as a product $\mathbf{D} \cong\left(\mathbf{D}_{1} \otimes_{\mathbb{Z}} \mathbb{Z}_{p}\right) \times \mathbf{D}_{2}$, where $\mathbf{D}_{1}$ is a $\mathbb{Z}$-root datum and $\mathbf{D}_{2}$ is a product of exotic $\mathbb{Z}_{p}$-root data.

2. Exotic $\mathbb{Z}_{p}$-root data are in 1-1 correspondence with exotic $\mathbb{Q}_{p}$-reflection groups via $\mathbf{D}=\left(W, L,\left\{\mathbb{Z}_{p} b_{\sigma}\right\}\right) \rightsquigarrow\left(W, L \otimes_{\mathbb{Z}_{p}} \mathbb{Q}_{p}\right)$.

Define the fundamental group as $\pi_{1}(\mathbf{D})=L / L_{0}$, where $L_{0}=\sum_{\sigma} \mathbb{Z}_{p} b_{\sigma}$ is the coroot lattice, and likewise, with $p$-discrete torus $\breve{T}=L \otimes \mathbb{Z} / p^{\infty}$, we define the $p^{-}$ discrete center as $\breve{\mathcal{Z}}(\mathbf{D})=\bigcap_{\sigma} \operatorname{ker}\left(\breve{\beta}_{\sigma}: \breve{T} \rightarrow \mathbb{Z} / p^{\infty}\right)$; compare e.g. [15]. It turns out that $\pi_{1}(\mathbf{D})=\breve{\mathcal{Z}}(\mathbf{D})=0$ for all exotic root data, and this plays a role in the proof of the above statement. If $A$ is a finite subgroup of $\breve{\mathcal{Z}}(\mathbf{D})$, we can define a quotient root datum $\mathbf{D} / A$ by taking $\breve{T}_{\mathbf{D} / A}=\breve{T} / A$, and hence $L_{\mathbf{D} / A}=\operatorname{Hom}\left(\mathbb{Z} / p^{\infty}, \breve{T} / A\right)$, and defining the roots and coroots of $\mathbf{D} / A$ via the induced maps.

Theorem 1.3 (The classification of $\mathbb{Z}_{p}$-root data, structure version).

1. Any $\mathbb{Z}_{p}$-root datum $\mathbf{D}=\left(W, L,\left\{\mathbb{Z}_{p} b_{\sigma}\right\}\right)$ can be written as a quotient

$$
\mathbf{D}=\left(\mathbf{D}_{1} \times \cdots \times \mathbf{D}_{n} \times\left(1, L^{W}, \emptyset\right)\right) / A
$$

where $\pi_{1}\left(\mathbf{D}_{i}\right)=0$ for all $i$, for a finite central subgroup $A$.

2. Irreducible $\mathbb{Z}_{p}$-root data $\mathbf{D}$ with $\pi_{1}(\mathbf{D})=0$ are in $1-1$ or $2-1$ correspondence with non-trivial irreducible $\mathbb{Q}_{p}$-reflection groups via $\mathbf{D} \rightsquigarrow\left(W, L \otimes_{\mathbb{Z}_{p}} \mathbb{Q}_{p}\right)$, the sole identification being $\mathbf{D}_{\mathrm{Sp}(n)} \otimes_{\mathbb{Z}} \mathbb{Z}_{2}$ with $\mathbf{D}_{\mathrm{Spin}(2 n+1)} \otimes_{\mathbb{Z}} \mathbb{Z}_{2}, n \geq 3$.

A main ingredient used to derive the classification of root data from the classification of $\mathbb{Q}_{p}$-reflection groups is the case-by-case observation that the $\bmod p$ reduction of all the exotic reflection groups remain irreducible, which ensures that any lift to $\mathbb{Z}_{p}$ is uniquely determined by the $\mathbb{Q}_{p}-$ representation.

Remark 1.4. It seems that $\mathbb{Z}_{p}$-root data ought to parametrize some purely algebraic objects, just as $\mathbb{Z}$-root data parametrize both compact connected Lie groups and reductive algebraic groups. Similar structures come up in Lusztig's approach to the representation theory of finite groups of Lie type, as examined by Bessis, Broué, Malle, Michel, Rouquier, and others [23, involving mythical objects from the Greek island of Spetses 68 . 


\section{2. $p$-compact groups and their classification}

In this section we give a brief introduction to $p$-compact groups, followed by the statement of the classification theorem, an outline of its proof, and some of its consequences. Additional background information on $p$-compact groups can be found in the surveys [36, 65, 72, 78].

The first ingredient we need is the theory of $p$-completions. The $p$-completion construction of Sullivan [95] produces for each space $X$ a map $X \rightarrow X_{p}$, which, when $X$ is simply connected and of finite type, has the property that $\pi_{i}\left(X_{p}\right) \cong$ $\pi_{i}(X) \otimes \mathbb{Z}_{p}$ for all $i$. A space is called $p$-complete if this map is a homotopy equivalence. In fact, when $X$ is simply connected and $H_{*}\left(X ; \mathbb{F}_{p}\right)$ is of finite type, then $X$ is $p$-complete if and only if the homotopy groups of $X$ are finitely generated $\mathbb{Z}_{p}$-modules. We remark that Bousfield-Kan [16] produced a variant on Sullivan's $p$-completion functor, and for the spaces that occur in this paper these two constructions agree up to homotopy, so the words $p$-complete and $p$-completion can be taken in either sense.

A finite loop space is a triple $(X, B X, e)$, where $B X$ is a pointed connected space, $X$ is a finite CW-complex, and $e: X \rightarrow \Omega B X$ is a homotopy equivalence, where $\Omega$ denotes based loops. We will return to finite loop spaces in Section 3 , but now move straight to their $p$-complete analogs.

Definition 2.1 ( $p$-compact group [42]). A p-compact group is a triple $(X, B X, e)$, where $B X$ is a pointed, connected, p-complete space, $H^{*}\left(X ; \mathbb{F}_{p}\right)$ is finite, and $e: X \stackrel{\simeq}{\rightarrow} \Omega B X$ is a homotopy equivalence.

The loop multiplication on $\Omega B X$ is here the homotopical analog of a group structure; while standard loop multiplication does not define a group, it is equivalent in a strong sense (as an $A_{\infty}$-space) to a topological group, whose classifying space is homotopy equivalent to $B X$. We therefore baptise $B X$ the classifying space, and note that, since all structure can be derived from $B X$, one could equivalently have defined a $p$-compact group to be a space $B X$, subject to the above conditions. The finiteness of $H^{*}\left(X ; \mathbb{F}_{p}\right)$ is to be thought of as a homotopical version of compactness, and replaces the condition that the underlying loop space be homotopy equivalent to a finite complex. We will usually refer to a $p$-compact group just by $X$ or $B X$ when there is little possibility for confusion.

Examples of $p$-compact groups include of course the $p$-completed classifying space $B G_{p}^{\wedge}$ of a compact Lie group $G$ with $\pi_{0}(G)$ a $p$-group. However, nonisomorphic compact Lie groups may give rise to equivalent $p$-compact groups if they have the same $p$-local structure, perhaps the most interesting example being $B \operatorname{SO}(2 n+1) \hat{p} \simeq B \operatorname{Sp}(n) \hat{p}$ for $p$ odd [46]. Exotic examples (i.e. examples with exotic root data) are discussed in Section 2.1 .

A morphism between $p$-compact groups is a pointed map $B X \rightarrow B Y$; it is called a monomorphism if the homotopy fiber, denoted $Y / X$, has finite $\mathbb{F}_{p^{-}}$ homology. Two morphisms are called conjugate if they are freely homotopic, and two $p$-compact groups are called isomorphic if their classifying spaces are homotopy equivalent. A $p$-compact group is called connected if $X$ is connected. By a 
standard argument $H^{*}\left(B X ; \mathbb{Z}_{p}\right) \otimes \mathbb{Q}$ is seen to be a $\mathbb{Q}_{p}-$ polynomial algebra, and we define the rank $r=\operatorname{rank}(X)$ to be number of generators. The following is the main structural result of Dwyer-Wilkerson [42].

Theorem 2.2 (Maximal tori and Weyl groups of $p$-compact groups 42]).

1. Any p-compact group $X$ has a maximal torus: a monomorphism $i: B T=$ $\left(B S^{1} \hat{p}\right)^{r} \rightarrow B X$ with $r$ the rank of $X$. Any other monomorphism $i^{\prime}: B T^{\prime}=$ $\left(B S^{1} \hat{p}\right)^{s} \rightarrow B X$ factors as $i^{\prime} \simeq i \circ \varphi$ for some $\varphi: B T^{\prime} \rightarrow B T$. In particular $i$ is unique up to conjugacy.

2. The Weyl space $\mathcal{W}_{X}(T)$, defined as the topological monoid of self-equivalences $B T \rightarrow B T$ over $i$ (with $i$ made into a fibration), has contractible components.

3. If $X$ is connected, the natural action of the Weyl group $W_{X}(T)=\pi_{0}\left(\mathcal{W}_{X}(T)\right)$ on $L_{X}=\pi_{2}(B T)$ gives a faithful representation of $W_{X}$ as a finite $\mathbb{Z}_{p}{ }^{-}$ reflection group.

A short outline of the proof can be found in [65]. The maximal torus normalizer is defined as the homotopy orbit space, or Borel construction, $B \mathcal{N}_{X}(T)=$ $B T_{h \mathcal{W}_{X}(T)}$ and hence sits in a fibration sequence

$$
B T \rightarrow B \mathcal{N}_{X}(T) \rightarrow B \mathcal{W}_{X}(T) .
$$

The normalizer is said to be split if the above fibration has a section. It is worth mentioning that one sees that $\left(W_{X}, L_{X}\right)$ is a $\mathbb{Z}_{p}$-reflection group indirectly, by proving that

$$
H^{*}\left(B X ; \mathbb{Z}_{p}\right) \otimes \mathbb{Q} \cong\left(H^{*}\left(B T ; \mathbb{Z}_{p}\right) \otimes \mathbb{Q}\right)^{W_{X}}
$$

To define the $\mathbb{Z}_{p}$-root datum, one therefore needs to proceed differently [45, 6. 8. For $p$ odd, the $\mathbb{Z}_{p}$-root datum $\mathbf{D}_{X}$ can be defined from the $\mathbb{Z}_{p}$-reflection group $\left(W_{X}, L_{X}\right)$, by setting $\mathbb{Z}_{p} b_{\sigma}=\operatorname{im}\left(L_{X} \stackrel{1-\sigma}{\longrightarrow} L_{X}\right)$. The definition for $p=2$ is slightly more complicated, and in order to give meaning to the words we first need a few extra definitions for $p$-compact groups. The centralizer of a morphism $\nu: B A \rightarrow B X$ is defined as $B C_{X}(\nu)=\operatorname{map}(B A, B X)_{\nu}$, where the subscript means the component corresponding to $\nu$. While this may look odd at first sight, it does in fact generalize the Lie group notion [35. For a connected $p$-compact group $X$ define the derived $p$-compact group $\mathcal{D} X$ to be the covering space of $X$ corresponding to the torsion subgroup of $\pi_{1}(X)$. Consider the $p$-discrete singular torus $\breve{T}_{0}^{\langle\sigma\rangle}$ for $\sigma$, i.e., the largest divisible subgroup of the fixed-points $\breve{T}^{\langle\sigma\rangle}$, with $\breve{T}=L_{X} \otimes \mathbb{Z} / p^{\infty}$, and set $X_{\sigma}=\mathcal{D}\left(\mathcal{C}_{X}\left(\breve{T}_{0}^{\langle\sigma\rangle}\right)\right)$. Then $X_{\sigma}$ is a connected $p$-compact group of rank one with $p$-discrete maximal torus $(1-\sigma) \breve{T}$; denote the corresponding maximal torus normalizer by $\mathcal{N}_{\sigma}$, called the root subgroup of $\sigma$. Define the coroots via the formula

$$
\mathbb{Z}_{p} b_{\sigma}= \begin{cases}\operatorname{im}\left(L_{X} \stackrel{1-\sigma}{\longrightarrow} L_{X}\right) & \text { if } \mathcal{N}_{\sigma} \text { is split } \\ \operatorname{ker}\left(L_{X} \stackrel{1+\sigma}{\longrightarrow} L_{X}\right) & \text { if } \mathcal{N}_{\sigma} \text { is not split }\end{cases}
$$

Only the first case happens for $p$ odd, and for $p=2$ the split case corresponds to $B X_{\sigma} \simeq B \mathrm{SO}(3)_{2}$ and the non-split corresponds to $B X_{\sigma} \simeq B \mathrm{SU}(2) \hat{2}$. For 
comparison we note that when $X=G_{p}$, for a reductive complex algebraic group $G$, $B X_{\sigma} \simeq B\left\langle U_{\alpha}, U_{-\alpha}\right\rangle_{p}$, where $U_{\alpha}$ is what is ordinarily called the root subgroup of the root $\alpha=\beta_{\sigma}$, and the above formula can be read off from e.g. [90, Pf. of Lem. 7.3.5].

We can now state the classification theorem.

Theorem 2.3 (Classification of $p$-compact groups [9, 8]). The assignment which to a connected $p$-compact group $X$ associates its $\mathbb{Z}_{p}$-root datum $\mathbf{D}_{X}$ gives a oneto-one correspondence between connected p-compact groups, up to isomorphism, and $\mathbb{Z}_{p}$-root data, up to isomorphism.

Furthermore the map $\Phi: \operatorname{Out}(B X) \rightarrow \operatorname{Out}\left(\mathbf{D}_{X}\right)$, given by lifting a self-homotopy equivalence of $B X$ to $B T$, is an isomorphism.

Here $\operatorname{Out}(B X)$ denotes the group of free homotopy classes of self-homotopy equivalences $B X \rightarrow B X$, and $\operatorname{Out}\left(\mathbf{D}_{X}\right)=\operatorname{Aut}\left(\mathbf{D}_{X}\right) / W_{X}$. A stronger space-level statement about self-maps is in fact true, namely

$$
B \operatorname{Aut}(B X) \stackrel{\simeq}{\rightarrow}\left(\left(B^{2} \breve{\mathcal{Z}}\left(\mathbf{D}_{X}\right)\right)_{p}\right)_{h} \operatorname{Out}\left(\mathbf{D}_{X}\right)
$$

where $\operatorname{Aut}(B X)$ is the space of self-homotopy equivalences, $\breve{\mathcal{Z}}\left(\mathbf{D}_{X}\right)$ the $p$-discrete center of $\mathbf{D}_{X}$ as introduced in Section[1, and the action of $\operatorname{Out}\left(\mathbf{D}_{X}\right)$ on $\left(B^{2} \breve{\mathcal{Z}}\left(\mathbf{D}_{X}\right)\right)_{p}$ is the canonical one. Having control of the whole space of self-equivalences turns out to be important in the proof.

Theorem 2.3 implies, by Theorem [1.2, that any connected $p$-compact group splits as a product of the $p$-completion of a compact connected Lie group and a product of known exotic $p$-compact groups. For $p=2$ it shows that there is only one exotic 2-compact group, the one corresponding to the $\mathbb{Q}_{2}$-reflection group $G_{24}$, and this 2-compact group was constructed in [41. We will return to the construction of the exotic $p$-compact groups in the next subsection.

Since we understand the whole space of self-equivalences, one can derive a classification also of non-connected $p$-compact groups. The set of isomorphism classes of non-connected $p$-compact groups with root datum of the identity component $\mathbf{D}$ and group of components $\pi$, is parametrized by the components of the moduli space

$$
\operatorname{map}\left(B \pi,\left(\left(B^{2} \breve{\mathcal{Z}}(\mathbf{D})\right)_{p}\right)_{h} \text { Out }(\mathbf{D})\right)_{h} \operatorname{Aut}(B \pi)
$$

As with the classification of compact Lie groups, the classification statement can naturally be broken up into two parts, existence and uniqueness of $p$-compact groups. The uniqueness statement can be formulated as an isomorphism theorem saying that there is a 1-1-correspondence between conjugacy classes of isomorphisms of connected $p$-compact groups $B X \rightarrow B X^{\prime}$ and isomorphisms of root data $\mathbf{D}_{X} \rightarrow \mathbf{D}_{X^{\prime}}$, up to $W_{X^{\prime}}$-conjugation. This last statement can in fact be strengthened to an isogeny theorem classifying maps that are rational isomorphisms [5].

While the existence and uniqueness are separate statements, they are currently most succinctly proved simultaneously by an induction on the size of $\mathbf{D}$, since the proof of existence requires knowledge of certain facts about self-maps, and the proof of uniqueness at the last step is aided by specific facts about concrete models. We will discuss the proof of existence in Section 2.1 and of uniqueness in Section 2.2. along with some information about the history. 
2.1. Construction of $\boldsymbol{p}$-compact groups. Compact connected Lie groups can be constructed in different ways. They can be exhibited as symmetries of geometric objects, or can be systematically constructed via generators-andrelations type constructions that involve first constructing a finite dimensional Lie algebra from the root system, and then passing to the group [61, 90].

An adaptation of the above tools to $p$-compact groups is still largely missing, so one currently has to proceed by more ad hoc means, with the limited aim of constructing only the exotic $p$-compact groups. These were in fact already constructed some years ago, but we take the opportunity here to retell the tale, and outline the closest we currently get to a streamlined construction.

The first exotic $p$-compact groups were constructed by Sullivan [95] as the homotopy orbit space of the action of the would-be Weyl group on the would-be torus. The most basic case he observed is the following: If $C_{m}$ is a cyclic group of order $m$, and $p$ an odd prime such that $m \mid p-1$, then $C_{m} \leq \mathbb{Z}_{p}^{\times}$, and hence $C_{m}$ acts on the Eilenberg-MacLane space $K\left(\mathbb{Z}_{p}, 2\right)$. The Serre spectral sequence for the fibration

$$
K\left(\mathbb{Z}_{p}, 2\right) \rightarrow K\left(\mathbb{Z}_{p}, 2\right)_{h C_{m}} \rightarrow B C_{m}
$$

reveals that the $\mathbb{F}_{p}$-cohomology of $K\left(\mathbb{Z}_{p}, 2\right)_{h C_{m}}$ is a polynomial algebra on a class in degree $2 m$, using that $m$ is prime to $p$. Therefore the cohomology of its loop space is an exterior algebra in degree $2 m-1$ and $B X=\left(K\left(\mathbb{Z}_{p}, 2\right)_{h C_{m}}\right) \hat{p}$ is a $p$ compact group, with $\Omega B X \simeq\left(S^{2 m-1}\right) \hat{p}$. We have just realized all exotic groups in family 3 of Table 1 !

Exactly the same argument carries over to the general case of a root datum D where $p \nmid|W|$, just replacing $C_{n}$ by $W$ and $\mathbb{Z}_{p}$ by $L$, since $\mathbb{F}_{p}\left[L \otimes \mathbb{F}_{p}\right]^{W}$ is a polynomial algebra exactly when $W$ is a reflection group, when $p \nmid|W|$, by the Shephard-Todd-Chevalley theorem used earlier. This observation was made by Clark-Ewing [31, and realizes a large number of groups in Table 1. However, the method as it stands cannot be pushed further, since the assumption that $p \nmid|W|$ is crucial for the collapse of the Serre spectral sequence.

Additional exotic $p$-compact groups were constructed in the 1970s by other methods. Quillen realized $G(m, 1, n)$ at all possible primes by constructing an approximation via classifying spaces of discrete groups [84, §10], and Zabrodsky [101, 4.3] realized $G_{12}$ and $G_{31}$ at $p=3$ and 5 respectively, by taking homotopy fixed-points of a $p^{\prime}$-group acting on the classifying space of a compact Lie group.

To build the remaining exotic $p$-compact groups one needs a far-reaching generalization of Sullivan's technique, obtained by replacing the homotopy orbit space with a more sophisticated homotopy colimit, that ensures that we still get a collapsing spectral sequence even when $p$ divides the order of $W$. The technique was introduced by Jackowski-McClure [54, as a decomposition technique in terms of centralizers of elementary abelian subgroups, and was subsequently used by Aguadé [2] $\left(G_{12}, G_{29}, G_{31}, G_{34}\right)$, Dwyer-Wilkerson [41] $\left(G_{24}\right)$, and Notbohm-Oliver 80. $(G(m, s, n))$ to finish the construction of the exotic $p$-compact groups.

The following is an extension of Aguadé's argument, and can be used inductively to realize all exotic $p$-compact groups for $p$ odd - that this works in all cases relies on the stroke of luck, checked case-by-case, that all exotic finite $\mathbb{Z}_{p}$-reflection 
groups for $p$ odd have $\mathbb{Z}_{p}[L]^{W}$ a polynomial algebra.

Theorem 2.4 (Inductive construction of $p$-compact groups, $p$ odd [9]). Consider a finite $\mathbb{Z}_{p}$-reflection group $(W, L)$, p odd, with $\mathbb{Z}_{p}[L]^{W}$ a polynomial algebra.

Then $\left(W_{V}, L\right)$ is a again a $\mathbb{Z}_{p}$-reflection group and $\mathbb{Z}_{p}[L]^{W_{V}}$ a polynomial algebra, for $W_{V}$ the pointwise stabilizer in $W$ of $V \leq L \otimes \mathbb{F}_{p}$.

Assume that, for all non-trivial $V,\left(W_{V}, L\right)$ is realized by a connected $p$-compact group $Y_{V}$ satisfying the isomorphism part of Theorem 2.3 and $H^{*}\left(Y_{V} ; \mathbb{Z}_{p}\right) \cong$ $\mathbb{Z}_{p}[L]^{W_{V}}$ (with $L$ in degree 2). Then $V \mapsto Y_{V}$ extends to a functor $Y: \mathbf{A}^{o p} \rightarrow$ Spaces, where $\mathbf{A}$ has objects non-trivial $V \leq L \otimes \mathbb{F}_{p}$ and morphisms given by conjugation in $W$, and

$$
B X=\left(\operatorname{hocolim}_{\mathbf{A}}{ }^{o p} Y\right) \hat{p}
$$

is a p-compact group with Weyl group $(W, L)$ and $H^{*}\left(B X ; \mathbb{Z}_{p}\right) \cong \mathbb{Z}_{p}[L]^{W}$.

Idea of proof. The statement that $\mathbb{Z}_{p}[L]^{W_{V}}$ is a polynomial algebra is an extension of Steinberg's fixed-point theorem in the version of Nakajima [75, Lem. 1.4]. The proof uses Lannes' $T$-functor, together with case-by-case considerations.

The inductive construction is straightforward, given current technology, and uses only general arguments: Since we assume we know $Y_{V}$ and its automorphisms for all $V \neq 1$, one easily sets up a functor $\mathbf{A}^{o p} \rightarrow \mathrm{Ho}($ Spaces), the homotopy category of spaces, and the task is to rigidify this to a functor in the category of spaces. The diagram can be show to be "centric", so one can use the obstruction theory developed by Dwyer-Kan in [37. The relevant obstruction groups identify with the higher limits of a functor obtained by taking fixed-points, and in particular form a Mackey functor whose higher limits vanish by a theorem of JackowskiMcClure 54. We can therefore rigidify the diagram to a diagram in spaces, and the resulting homotopy colimit is easily shown to have the desired cohomology.

We now turn to the prime 2 . Here the sole exotic $\mathbb{Z}_{2}$-reflection group is $G_{24}$, and the corresponding 2-compact group was realized by Dwyer-Wilkerson [4] and dubbed DI(4), due to the fact that, for $E=(\mathbb{Z} / 2)^{4}$,

$$
H^{*}\left(B \mathrm{DI}(4) ; \mathbb{F}_{2}\right) \cong \mathbb{F}_{2}[E]^{\mathrm{GL}(E)}
$$

the rank four Dickson invariants. At first glance this might look like the setup of Theorem 2.4, but note that $G_{24}$ is a rank three $\mathbb{Z}_{2}$-reflection group, not four, so $E$ is not just the elements of order 2 in the maximal torus. However by taking $\mathbf{A}$ to be the category with objects the non-trivial subgroups of $E$, and morphisms induced by conjugation in $\mathrm{GL}(E)$, and correctly guessing the centralizers of elementary abelian subgroups, the argument can still be pushed through; the starting point is declaring the centralizer of any element of order two to be $\operatorname{Spin}(7) \hat{2}$.

We again stress the apparent luck in being able to guess the rather uncomplicated structure of $\mathbf{A}$ and the centralizers. If one hypothetically had to construct an exotic $p$-compact group with a seriously complicated cohomology ring, say one would try to construct $E_{8}$ at the prime 2 by these methods, it would not be clear how to start. As a first step one would need a way to describe the $p$-fusion in the group, just from the root datum D. This relates to old questions in Lie theory, which have occupied Borel, Serre, and many others [88]... 
2.2. Uniqueness of $\boldsymbol{p}$-compact groups. In this subsection, we outline the proof of the uniqueness part of the classification theorem for $p$-compact groups, Theorem [2.3, following [8] by Andersen and the author; it extends [9] also with Møller and Viruel. We mention that the quest for uniqueness was initiated by Dwyer-Miller-Wilkerson [38] in the 80s and in particular Notbohm 77] obtained strong partial results; a different approach for $p=2$ using computer algebra was independently given by Møller 73,74 . See $[9,8$, for more details on the history of the proof.

From now on we consider two connected $p$-compact groups $X$ and $X^{\prime}$ with the same root datum $\mathbf{D}$, and want to build a homotopy equivalence $B X \rightarrow B X^{\prime}$. The proof goes by an induction on the size of $(W, L)$.

Step 1: (The maximal torus normalizer and its automorphisms, [45, 6]). A first step is to show that $X$ and $X^{\prime}$ have isomorphic maximal torus normalizers. Working with the maximal torus normalizer has a number of technical advantages over the maximal torus, related to the fact that the fiber of the map $B \mathcal{N} \rightarrow B X$ has Euler characteristic prime to $p$ (one, actually).

One shows that the maximal torus normalizers are isomorphic, by giving a construction from the root datum. For $p$ odd the construction is simple, since the maximal torus normalizer turns out always to be split, and hence isomorphic to $\left(B^{2} L\right)_{h W}$ with the canonical action. This was established in [3], by showing that the relevant extension group is zero except in one case, which can be handled by other means; cf. also [9, Rem. 2.5]. For $p=2$, the problem is more difficult. The corresponding problem for compact Lie groups, or reductive algebraic groups, was solved by Tits [96 many years ago. A thorough reading of Tits' paper, with a cohomological rephrasing of some of his key constructions, allows his construction to be pushed through also for $p$-compact groups 45 . One thus algebraically constructs a maximal torus normalizer $\mathcal{N}_{\mathbf{D}}$ and show it to be isomorphic to the topologically defined one. A problem is however that $\mathcal{N}$ in general has too many automorphisms. To correct this, it was shown in [6] that the root subgroups $\mathcal{N}_{\sigma}$, introduced before Theorem 2.3. can also be built algebraically, and adding this extra data give the correct automorphisms. Concretely, one has a canonical factorization

$$
\Phi: \operatorname{Out}(B X) \rightarrow \operatorname{Out}\left(B \mathcal{N},\left\{B \mathcal{N}_{\sigma}\right\}\right) \stackrel{\cong}{ } \operatorname{Out}\left(\mathbf{D}_{X}\right)
$$

and one can furthermore build a candidate model for the whole space $B \operatorname{Aut}(B X)$, by a slight modification of $B \operatorname{Aut}\left(B \mathcal{N},\left\{B \mathcal{N}_{\sigma}\right\}\right)$, the space of self-homotopy equivalences of $B \mathcal{N}$ preserving the root subgroups.

Step 2: (Reduction to simple, center-free groups, [8, §2]). This next step involves relating the $p$-compact group and its summands and center-free quotient via certain fibration sequences, and studying automorphisms via these fibrations. Several of the necessary tools, such as the understanding of the center of a $p$-compact group [43, the product splitting theorem [44], etc., were already available in the $90 \mathrm{~s}$. But, in particular for $p=2$, one needs to incorporate the machinery of root data and root subgroups; we refer the reader to [, §2] for the details. 
Step 3: (Defining a map on centralizers of elements of order $p$, [8, §4]). We now assume that $X$ and $X^{\prime}$ are simple, center-free $p$-compact groups. The next tool needed is a homology decomposition theorem, more precisely the centralizer decomposition, of Jackowski-McClure [54] and Dwyer-Wilkerson [40], already mentioned in the previous subsection. Let $\mathbf{A}(X)$ be the Quillen category of $X$ with objects monomorphisms $\nu: B E \rightarrow B X$, where $E=(\mathbb{Z} / p)^{s}$ is a non-trivial elementary abelian $p$-group, and the morphisms $(\nu: B E \rightarrow B X) \rightarrow\left(\nu^{\prime}: B E^{\prime} \rightarrow B X\right)$ are the group monomorphisms $\varphi: E \rightarrow E^{\prime}$ such that $\nu^{\prime} \circ B \varphi$ is conjugate to $\nu$. The centralizer decomposition theorem now says that for any $p$-compact group $X$, the evaluation map

$$
\operatorname{hocolim}_{\nu \in \mathbf{A}(X)^{\text {op }}} B \mathcal{C}_{X}(\nu) \rightarrow B X
$$

is an isomorphism on $\mathbb{F}_{p}$-cohomology.

This opens the possibility for a proof by induction, since the centralizers will be smaller $p$-compact groups if $X$ is center-free. As explained above we can assume that $X$ and $X^{\prime}$ have common maximal torus normalizer and root subgroups $\left(\mathcal{N},\left\{\mathcal{N}_{\sigma}\right\}\right)$, so that we are in the situation of following diagram

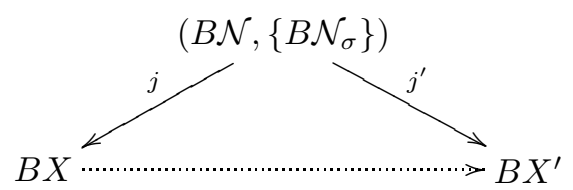

where the dotted arrow is the one we want to construct.

If $\nu: B \mathbb{Z} / p \rightarrow B X$ is a monomorphism, then it can be conjugated into $T$, uniquely up to conjugation in $\mathcal{N}$. This gives a well defined way of viewing $\nu$ as a map $\nu: B \mathbb{Z} / p \rightarrow B T \rightarrow B \mathcal{N}$. Taking centralizers of this map produces a new diagram

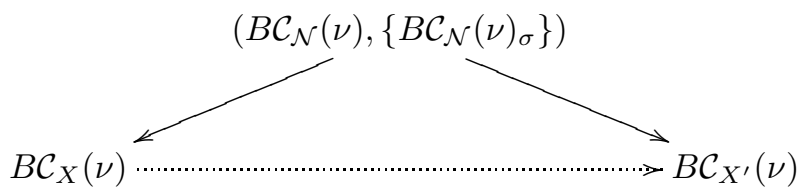

One now argues that the induction hypothesis guarantees that we can construct the dotted arrow. There is the slight twist that the centralizer will be disconnected in general, so we have to use that we inductively know the whole space of selfequivalences of the identity component.

Step 4: (Compatibility of maps on all centralizers, [8, §5]). The next step is to define the map on centralizers of arbitrary elementary abelian $p$-subgroups $\nu: B E \rightarrow B X$. This is done by restricting to a rank one subgroup $E^{\prime} \leq E$ and considering the composition

$$
B \mathcal{C}_{X}(\nu) \rightarrow B \mathcal{C}_{X}\left(\left.\nu\right|_{E^{\prime}}\right) \rightarrow B \mathcal{C}_{X^{\prime}}\left(\left.\nu\right|_{E^{\prime}}\right) \rightarrow B X^{\prime} .
$$

One now has to show that these maps do not depend on the choice of $E^{\prime}$, and that they fit together to define an element in

$$
\lim _{\nu \in \mathbf{A}(X)}{ }^{0}\left[B \mathcal{C}_{X}(\nu), B X^{\prime}\right]
$$


By using induction it turns out that one can reduce to the case where $E$ has rank two and $\mathcal{C}_{X}(\nu)$ is discrete. An inspection of the classification of $\mathbb{Z}_{p}$-root data shows

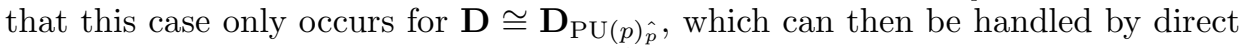
arguments, producing the element in $\lim ^{0}$.

In fact one can prove something slightly stronger, which will be needed in the next step: A close inspection of the whole preceeding argument reveals that all maps can be constructed over $B^{2} \pi_{1}(\mathbf{D})$, which allows one to produce an element in

$$
\lim _{\nu \in \mathbf{A}(X)}{ }^{0}\left[\widehat{B \mathcal{C}_{X}(\nu)}, B \widetilde{X^{\prime}}\right]
$$

where the tilde denotes covers with respect to the kernel of the map to $\pi_{1}(\mathbf{D})$.

With this step complete one can see that $B X$ and $B X^{\prime}$ have the same $p$ fusion, i.e., that $p$-subgroups are conjugate in the same way, but we are left with a rigidification issue.

Step 5: (Rigidifying the map, [8, $\S 6]$ ). One now wants to define a map on the whole homotopy colimit, which can then easily be checked to have the correct properties, finishing the proof of the classification. Constructing such a map directly from an element in $\lim ^{0}$ requires knowing that the higher limits of the functors $F_{i}: \mathbf{A}(X) \rightarrow$ $\mathbb{Z}_{p^{-}}$mod given by $E \mapsto \pi_{i}\left(\mathcal{Z C}_{X}(E)\right)$, vanish, where $\mathcal{Z}$ denotes the center. In turn, this calculation requires knowing the structure of $\mathbf{A}(X)$, and for this we use that $X$ is a known $p$-compact group, where we can examine the structure. For the part of the functor corresponding to elementary abelian subgroups that can be conjugated into $T$, the higher limits can be show to vanish via a Mackey functor argument, going back to [54] and [40]. This in fact equals the whole functor for all exotic groups for $p$ odd, and DI(4) also works via a variant on this argument, which finish off those cases.

We can hence assume that $X$ is the $p$-completion of a compact connected Lie group $G$. Here the obstruction groups were computed to identically vanish in [9], for $p$ odd, relying on detailed information about the elementary abelian $p$-subgroups of $G$, partially tabulated by Griess 48. This is easy when there is little torsion in the cohomology, but harder for the small torsion primes, and the exceptional groups. In [8], however, we use a different argument to cover all primes. Using the above element in $\lim ^{0}$ it turns out that one can produce an element in

$$
\lim _{\tilde{G} / \tilde{P} \in \mathbf{O}_{p}^{r}(\tilde{G})^{\text {op }}}\left[B \tilde{P}, B \widetilde{X^{\prime}}\right]
$$

where $\mathbf{O}_{p}^{r}(\tilde{G})$ is the subcategory of the orbit category of $G$ with objects the socalled $p$-radical subgroups. Here one again wants to show vanishing of the higher limits, in order to get a map on the homotopy colimit. Calculating higher limits over this orbit category is in many ways similar to calculating it over the Quillen category 49]. In this case, however, the relevant higher limits were in fact shown to identically vanish in earlier work of Jackowski-McClure-Oliver [55], also building on substantial case-by-case calculations. This again produces a map $B \tilde{G} \stackrel{\simeq}{\longrightarrow} B \widetilde{X^{\prime}}$, and passing to a quotient provides the sought homotopy equivalence $B G \stackrel{\simeq}{\longrightarrow} B X^{\prime}$. The statements about self-maps also fall out of this approach. 
2.3. Lie theory for $\boldsymbol{p}$-compact groups. We have already seen many Lie-type results for $p$-compact groups. Quite a few more can be proved by observing that the classical Lie result only depends on the $p$-completion of the compact Lie group, and verifying case-by-case that it holds for the exotic $p$-compact groups. We collect some theorems of this type in this section, encouraging the reader to look for more conceptual proofs, and include also a brief discussion of homotopical representation theory. Throughout this section $X$ is a connected $p$-compact group with maximal torus $T$.

The first theorem on the list is the analog of theorems of Bott [14] from 1954.

Theorem 2.5. $H^{*}\left(X / T ; \mathbb{Z}_{p}\right)$ and $H^{*}\left(\Omega X ; \mathbb{Z}_{p}\right)$ are both torsion free and concentrated in even degrees, and $H^{*}\left(X / T ; \mathbb{Z}_{p}\right)$ has rank $\left|W_{X}\right|$ as a $\mathbb{Z}_{p}$-module.

The result about $\Omega X$ was known as the loop space conjecture, and in fact proved by Lin and Kane in a series of papers in the more general setting of finite mod $p H$-spaces, using complicated calculations with Steenrod operations 67.

Bott's proof used Morse theory and the result may be viewed in the context of Schubert cell decompositions [1]. Rationally $H^{*}\left(X / T ; \mathbb{Z}_{p}\right) \otimes \mathbb{Q}=\mathbb{Q}_{p}[L] \otimes_{\mathbb{Q}_{p}[L]^{W_{X}}}$ $\mathbb{Q}_{p}$, so calculating the Betti numbers, given the theorem, is reduced to a question about complex reflection groups - an interpretation of these numbers in terms of length functions on the root system has been obtained for certain classes of complex reflection groups, cf. [17, 98, but the complete picture is still not clear. In general the theory of homogeneous and symmetric spaces for $p$-compact groups is rather unexplored, and warrants attention.

Theorem 2.5]implies that $\pi_{3}(X)$ is torsion free, and proving that in a conceptual way might be a good starting point. For Lie groups, Bott in fact stated the, now classical, fact that $\pi_{3}(G) \cong \mathbb{Z}$ for $G$ simple. The analogous statement is not true for most of the exotic $p$-compact groups; for instance it obviously fail for the Sullivan spheres other than $S^{3}$. However, it is true that $\pi_{3}$ is non-zero for finite loop spaces, as a consequence of a celebrated theorem of Clark 30 from 1963 giving strong restrictions on the degrees of finite loop spaces. These results helped fuel the speculation that finite loop spaces should look a lot like compact Lie groups, a point we will return to in the next section.

Most of the general results about torsion in the cohomology of $B X$ and $X$ due to Borel, Steinberg, and others, also carry through to $p$-compact groups, but here again with many results relying on the classification. This fault is partly inherited from Lie groups; see Borel [13, p. 775] for a summary of the status there. In particular we mention that $X$ has torsion free $\mathbb{Z}_{p}$-cohomology if and only if $B X$ has torsion free $\mathbb{Z}_{p}$-cohomology if and only if every elementary abelian $p$-subgroup factors through a maximal torus. Likewise $\pi_{1}(X)$ is torsion free if and only if every elementary abelian group of rank two factors through a maximal torus; see [9, 8.

The (complex linear) homotopy representation theory of $X$ is encoded in the semi-ring

$$
\operatorname{Rep}^{\mathbb{C}}(B X)=\left[B X, \coprod_{n} B \mathrm{U}(n)_{p}^{\hat{p}}\right]
$$


It is non-trivial since for any connected $p$-compact group $X$ there exists a monomorphism $B X \rightarrow B \mathrm{U}(n)_{p}$, for some $n$; the exotic groups were checked in 27, 28, 102 - indeed, as already alluded to, several exotic $p$-compact groups can conveniently be constructed as homotopy fixed-points inside a $p$-completed compact Lie group. The general structure of the semi-ring is however still far from understood. The classification allows one to focus on $p$-completed classifying spaces of compact Lie groups, but even in this case the semi-ring appears very complicated [56]; there are higher limits obstructions, related to interesting problems in group theory [49].

Weights can be constructed as usual: By the existence of a maximal torus, we can lift a homotopy representation to a map $B T_{X} \rightarrow B T_{\mathrm{U}(n)_{p}}$, well defined up to an action of $\Sigma_{n}$, and produce a collection of $n$ weights in $L_{X}^{*}=\operatorname{Hom}_{\mathbb{Z}_{p}}\left(L_{X}, \mathbb{Z}_{p}\right)$, invariant under the action of the Weyl group $W_{X}$. When $p \nmid\left|W_{X}\right|$, homotopy representations just correspond to finite $W_{X}$-invariant subsets of $L_{X}^{*}$, and any homotopy representation decomposes up to conjugation uniquely into indecomposable representations given by transitive $W_{X}$-sets. When $p|| W_{X} \mid$ the situation is much more complicated.

Let us describe what happens in the basic case of $X=\mathrm{SU}(2) \hat{2}$. Denote by $\rho_{i}$ the irreducible complex representation of $\mathrm{SU}(2)$ with highest weight $i$, and use the same letter for the induced map $B \mathrm{SU}(2) \hat{2} \rightarrow B \mathrm{U}(i+1) \hat{2}$. Precomposing with the self-homotopy equivalence $\psi^{k}$ of $B \mathrm{SU}(2) \hat{2}, k \in \mathbb{Z}_{2}^{\times}$, corresponding to multiplication by $k$ on the root datum, gives a new representation $k \star \rho_{i}$ of the same dimension, but with weights multiplied by $k$.

Theorem 2.6. $\operatorname{Rep}^{\mathbb{C}}(B \mathrm{SU}(2) \hat{2})$ has an additive generating set given by $\rho_{0}, k \star \rho_{1}$, $k \star \rho_{2}$ and $\left(\left(k+2 k^{\prime}\right) \star \rho_{1}\right) \otimes\left(\left(k-2 k^{\prime}\right) \star \rho_{1}\right), k \in \mathbb{Z}_{2}^{\times}, 0 \neq k^{\prime} \in \mathbb{Z}_{2}$. These generators are indecomposable, and two representations agree if they have the same weights.

The reader may verify that the decomposition into indecomposables is not unique, e.g. for $\rho_{6}$. It is at present not clear how to use $\mathrm{SU}(2) \hat{2}$ to describe the general structure, as one could have hoped - the thing to note is that homotopy representations are governed by questions of $p$-fusion of elements, rather than more global structure. Already for $\mathrm{SU}(2)_{2} \times \mathrm{SU}(2) \hat{2}$ there is no upper bound on the dimension of the indecomposables, and in particular they are not always a tensor product of indecomposable $\mathrm{SU}(2) \hat{2}$ representations. More severely, representations need not be uniquely determined by their weights, e.g. for $\operatorname{Sp}(2) \hat{2} \times \operatorname{Sp}(2) \hat{2}$.

By using case-by-case arguments, there might be hope to establish a version of Weyl's theorem $R(B X) \stackrel{\cong}{\rightarrow} R(B T)^{W_{X}}$, where $R(B X)=\operatorname{Gr}\left(\operatorname{Rep}^{\mathbf{C}}(B X)\right)$ is the Grothendieck group. The result is not proved even for $p$-completions of compact Lie groups, but the integral version is the main result in [58. The weaker $K_{-}$ theoretic result $K^{*}\left(B X ; \mathbb{Z}_{p}\right) \stackrel{\cong}{\rightrightarrows} K^{*}\left(B T ; \mathbb{Z}_{p}\right)^{W}$ was established in [60] (using that $H^{*}\left(\Omega X ; \mathbb{Z}_{p}\right)$ is torsion free). The ring structure of $R(B T)^{W}$ is also not clear, and in particular it would be interesting to exhibit some fundamental representations. 


\section{Finite loop spaces}

In the 1960s and early 1970s, finite loop spaces, not $p$-compact groups, were the primary objects of study, and there were many conjectures about them 91. The theory of $p$-compact groups enables the resolution of most of them, either in the positive or the negative, and gives what is essentially a parametrization of all finite loop spaces.

We already defined finite loop spaces in Section 2, let us now briefly recall their history in broad strokes. Hopf proved in 1941 [52 that the rational cohomology of any connected, finite loop space is a graded exterior algebra $H^{*}(X ; \mathbb{Q}) \cong$ $\bigwedge_{\mathbb{Q}}\left(x_{1}, \ldots, x_{r}\right)$, where $\left|x_{i}\right|=2 d_{i}-1$, and $r$ is called the rank. Serre, ten years later [87, showed that the list of degrees $d_{1}, \ldots, d_{r}$ uniquely determines the rational homotopy type of $(X, B X, e)$. In those days, there were not many examples of finite loop spaces. Indeed, in the early 1960 s it was speculated that perhaps every finite loop space was homotopy equivalent to a compact Lie group, a would-be variant of Hilbert's 5th problem. This was soon shown to be wrong in several different ways: Hilton-Roitberg, in 1968, exhibited a 'criminal' [51, a finite loop space $(X, B X, e)$, of the rational homotopy type of $\operatorname{Sp}(2)$, such that the underlying space $X$ is not homotopy equivalent to any Lie group; and Rector [85] in 1971 observed that there exists uncountable many finite loop spaces $(X, B X, e)$ such that $X$ is homotopy equivalent to $\mathrm{SU}(2)$. The first example may superficially look more benign than the second; indeed in general there are only finitely many possibilities for the homotopy type of the underlying space $X$, given the rational homotopy type of $B X$ 32. But the exact number depends on homotopy groups of finite complexes, and does not appear closely related to Lie theory, so shifting focus from loop space structures $(X, B X, e)$ to that of homotopy types of $X$, does not appear desirable.

An apparently better option is, as the reader has probably sensed, to pass to $p$-completions, defined in Section 2. Sullivan made precise how one can recover a (simply connected) space integrally if one knows the space "at all primes and rationally, as well as how they are glued together". Along with his $p$-completion, he constructed a rationalization functor $X \rightarrow X_{\mathbb{Q}}$, with analogous properties, and proved that these functors fit together in the following arithmetic square.

Proposition 3.1 (Sullivan's arithmetic square [94, 34]). Let $Y$ be a simply connected space of finite type. Then the following diagram, with obvious maps, is a homotopy pull-back square.

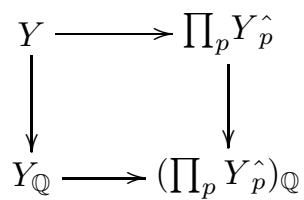

This parallels the usual fact that the integers $\mathbb{Z}$ is a pullback of $\hat{\mathbb{Z}}=\prod_{p} \mathbb{Z}_{p}$ and $\mathbb{Q}$ over the finite adeles $\mathbb{A}_{f}=\hat{\mathbb{Z}} \otimes \mathbb{Q}$. If $B X$ is the classifying space of a connected finite loop space then, by the classification of $p$-compact groups, all 
spaces in the diagram are now understood: Each $B X_{\hat{p}}$ is the classifying space of a $p$-compact group, and the spaces at the bottom of the diagram are determined by numerical data, namely the degrees: $B X_{\mathbb{Q}} \simeq K\left(\mathbb{Q}, 2 d_{1}\right) \times \cdots \times K\left(\mathbb{Q}, 2 d_{r}\right)$ and $\left(\prod_{p} B X_{p}^{\hat{p}}\right)_{\mathbb{Q}} \simeq K\left(\mathbb{A}_{f}, 2 d_{1}\right) \times \cdots \times K\left(\mathbb{A}_{f}, 2 d_{r}\right)$, by the result of Serre quoted earlier. Hence to classify finite loop spaces with some given degrees, we first have to enumerate all collections of $p$-compact groups with those degrees; there are a finite number of these, and they can be enumerated given the classification 8, Prop. 8.18]. The question of how many finite loop spaces with a given set of $p$ completions is then a question of genus, determined by an explicit set of double cosets.

Theorem 3.2 (Classification of finite loop spaces). The assignment which to a finite loop space $Y$ associates the collection of $\mathbb{Z}_{p}$-root data $\left\{\mathbf{D}_{Y_{\hat{p}}}\right\}_{p}$ is a surjection from connected finite loop spaces to collections of $\mathbb{Z}_{p}$-root data, all p, with the same degrees $d_{1}, \ldots, d_{r}$. The pre-image of $\left\{\mathbf{D}_{p}\right\}_{p}$ is parametrized by the set of double cosets

$$
\operatorname{Out}\left(K_{\mathbb{Q}}\right) \backslash \operatorname{Out}^{c}\left(K_{\mathbb{A}_{f}}\right) / \prod_{p} \operatorname{Out}\left(\mathbf{D}_{p}\right)
$$

where $K_{\mathbb{F}}=K\left(\mathbb{F}, 2 d_{1}\right) \times \cdots \times K\left(\mathbb{F}, 2 d_{r}\right), \mathbb{F}=\mathbb{Q}$ or $\mathbb{A}_{f}$.

Here $\operatorname{Out}\left(K_{\mathbb{Q}}\right)$ denotes the group of free homotopy classes of self-homotopy equivalences, and $\operatorname{Out}^{c}\left(K_{\mathbb{A}_{f}}\right)$ denotes those homotopy equivalences that induce $\mathbb{A}_{f}$-linear maps on homotopy groups. Since $K_{\mathbb{F}}$ is an Eilenberg-MacLane space, the set of double cosets can be completely described algebraically; see [9, §13] for a calculation of $\operatorname{Out}\left(\mathbf{D}_{p}\right)$.

The set of double cosets will, except for the degenerate case of tori, be uncountable. Allowing for only a single prime $p$ everywhere above would parametrize the number of $\mathbb{Z}_{(p)}$-local finite loop spaces corresponding to a given $p$-compact group $Y_{p}$, and also this set is usually uncountable, with a few more exceptions, such as $\mathrm{SU}(2)$. A similar result holds when one inverts some collection of primes $\mathcal{P}$; see [7, Rem. 3.3] for more information.

Sketch of proof of Theorem 3.2. There is a natural inclusion $K_{\mathbb{Q}} \rightarrow K_{\mathbb{A}_{f}}$ induced by the unit map $\mathbb{Q} \rightarrow \mathbb{A}_{f}$, and one easily proves that the pull-back provides a space $Y$ such that $H^{*}(\Omega Y ; \mathbb{Z})$ is finite over $\mathbb{Z}$. That $Y$ is actually homotopy equivalent to a finite complex follows by the vanishing of the finiteness obstruction, as proved by Notbohm [81] (see [4, Lemma 1.2] for more details). Twisting the pullback by an element in $\operatorname{Out}^{c}\left(K_{\mathbb{A}_{f}}\right)$ provides a new finite loop space, and after passing to sets of double cosets, this assignment is easily seen to be surjective and injective on homotopy types (see [94] and [100, Thm. 3.8]).

If one assumes that the finite loop space $X$ has a maximal torus, as defined by Rector [86, i.e., a map $\left(B S^{1}\right)^{r} \rightarrow B X$ with homotopy fiber homotopy equivalent to a finite complex, for $r=\operatorname{rank}(X)$, the above picture changes completely. The inclusion of an 'integral' maximal torus prohibits the twisting in the earlier theorem, and one obtains a proof of the classical maximal torus conjecture stated by 
Wilkerson [99] in 1974, giving a homotopy theoretical description of compact Lie groups as exactly the finite loop spaces admitting a maximal torus.

Theorem 3.3 (Maximal torus conjecture [8]). The classifying space functor, which to a compact Lie group $G$ associates the finite loop space $(G, B G, e: G \stackrel{\simeq}{\rightarrow} \Omega B G)$ gives a one-to-one correspondence between isomorphism classes of compact Lie groups and finite loop spaces with a maximal torus. Furthermore, for $G$ connected, $\operatorname{Out}(B G) \cong \operatorname{Out}(G) \cong \operatorname{Out}\left(\mathbf{D}_{G}\right)$.

The statement about automorphisms, which was not part of the original conjecture, follows from work of Jackowski-McClure-Oliver [57, Cor. 3.7].

In light of the above structural statement it is natural to further enquire how exotic finite loop spaces can be. Whether they are all manifolds was recently settled in the affirmative by Bauer-Kitchloo-Notbohm-Pedersen, answering an old question of Browder [24].

Theorem 3.4 (10]). For any finite loop space $(Y, B Y, e), Y$ is homotopy equivalent to a closed, smooth, parallelizable manifold.

The result is proved using the theory of $p$-compact groups, combined with classical surgery techniques, as set up by Pedersen. It shows the subtle failure of a naïve homotopical version of Hilbert's fifth problem: Every finite loop space is, by classical results, homotopy equivalent to a topological group, and homotopy equivalent to a compact smooth manifold by the above. But one cannot always achieve both properties at once. This would otherwise imply that every finite loop space was homotopy equivalent to a compact Lie group, by the solution to Hilbert's fifth problem, contradicting that many exotic finite loop spaces exist.

One can still ask if every finite loop space is rationally equivalent to some compact Lie group? Indeed this was conjectured in the 70s to be the case, and was verified up to rank 5. However, the answer to this question turns out to be negative as well, although counterexamples only start appearing in high rank.

Theorem 3.5 (A 'rational criminal' 4]). There exists a connected finite loop space $X$ of rank 66, dimension 1254, and degrees

$$
\left\{2^{8}, 3^{2}, 4^{8}, 5^{2}, 6^{7}, 7,8^{7}, 9,10^{5}, 11,12^{5}, 13,14^{5}, 16^{3}, 18^{2}, 20^{2}, 22,24^{2}, 26,28,30\right\}
$$

(where $n^{k}$ means that $n$ is repeated $k$ times) such that $H_{*}(X ; \mathbb{Q})$ does not agree with $H_{*}(G ; \mathbb{Q})$ for any compact Lie group $G$, as graded vector spaces.

This example is minimal, in the sense that any connected, finite loop space of rank less than 66 is rationally equivalent to some compact Lie group $G$.

In [4 there is a list of which $p$-compact group to choose at each prime. By the preceeding discussion, the problem of finding such a space is a combinatorial problem, and one can show that in high enough rank there will be many examples. 


\section{Steenrod's problem of realizing polynomial rings}

The 1960 "Steenrod problem" 92, 93, asks, for a given ring $R$, which graded polynomial algebras are realized as $H^{*}(Y ; R)$ of some space $Y$, i.e., in which degrees can the generators occur? In this section we give some background on this classical problem and describe its solution in [7, 8.

Steenrod, in his original paper 92 , addressed the case of polynomial rings in a single variable: For $R=\mathbb{Z}$ the only polynomial rings that occur are $H^{*}\left(\mathbb{C} P^{\infty} ; \mathbb{Z}\right) \cong$ $\mathbb{Z}\left[x_{2}\right]$ and $H^{*}\left(\mathbb{H} P^{\infty} ; \mathbb{Z}\right) \cong \mathbb{Z}\left[x_{4}\right]$, as he showed by a short argument using his cohomology operations. Similarly, for $R=\mathbb{F}_{p}$ he showed that the generator has to sit in degree 1,2 , or 4 for $p=2$ and in degree $2 n$ with $n \mid p-1$ for $p$ odd, but now as a consequence of Hopf invariant one and its odd primary version (though it was not known at the time whether the $p$ odd cases were realized when $n \neq 1,2$ ).

There were attempts to use the above techniques to settle polynomial rings in several variable, but they gave only very partial results. In the 70s, however, Sullivan's method, as generalized by Clark-Ewing, realized many polynomial rings, as explained in Section 2.1. Conversely, in the 80s Adams-Wilkerson [1] and others put restrictions on the potential degrees, using categorical properties of the category of unstable algebras over the Steenrod algebra. This eventually led to the result of Dwyer-Miller-Wilkerson [39 that for $p$ large enough the Clark-Ewing examples are exactly the possible polynomial cohomology rings over $\mathbb{F}_{p}$.

In order to tackle all primes, it turns out to be useful to have a space-level theory, and that is what $p$-compact groups provide. Namely, if $Y$ is a space such that $H^{*}\left(Y ; \mathbb{F}_{p}\right)$ is a polynomial algebra, then the Eilenberg-Moore spectral sequence shows that $H^{*}\left(\Omega Y ; \mathbb{F}_{p}\right)$ is finite, and hence $Y_{p}^{\hat{p}}$ is a $p$-compact group.

Theorem 4.1 (Steenrod's problem, $\operatorname{char}(R) \neq 2[7]$ ). Let $R$ be a commutative Noetherian ring of finite Krull dimension and let $P^{*}$ be a graded polynomial $R$ algebra in finitely many variables, all in positive even degrees.

Then there exists a space $Y$ such that $P^{*} \cong H^{*}(Y ; R)$ as graded algebras if and only if for each prime $p$ not a unit in $R$, the degrees of $P^{*}$ halved is a multiset union of the degrees lists occurring in Table 1 at that prime $p$, and the degree one, with the following exclusions (due to torsion): $(G(2,2, n), p=2) n \geq 4,(G(6,6,2), p=2)$, $\left(G_{24}, p=2\right),\left(G_{28}, p=2,3\right),\left(G_{35}, p=2,3\right),\left(G_{36}, p=2,3\right)$, and $\left(G_{37}, p=2,3,5\right)$.

When $\operatorname{char}(R) \neq 2$, all generators are in even degrees by anti-commutativity, so the assumptions of the theorem are satisfied. The proof in [7] only relies on the general theory of $p$-compact groups, not on the classification. The case $R=\mathbb{F}_{p}$, $p$ odd, was solved earlier by Notbohm [81, also using $p$-compact group theory. Taking $R=\mathbb{Z}$ gives the old conjecture that if $H^{*}(Y ; \mathbb{Z})$ is a polynomial ring, then it is isomorphic to a tensor product of copies of $\mathbb{Z}\left[x_{2}\right], \mathbb{Z}\left[x_{4}, x_{6}, \ldots, x_{2 n}\right]$, and $\mathbb{Z}\left[x_{4}, x_{8}, \ldots, x_{4 n}\right]$, the cohomology rings of $\mathbb{C} P^{\infty}, B \operatorname{SU}(n)$ and $B \operatorname{Sp}(n)$.

Theorem 4.2 (Steenrod's problem, $\operatorname{char}(R)=2$ [8]). Suppose that $P^{*}$ is a graded polynomial algebra in finitely many variables over a commutative ring $R$ of characteristic 2 . Then $P^{*} \cong H^{*}(Y ; R)$ for a space $Y$ if and only if

$$
P^{*} \cong H^{*}(B G ; R) \otimes H^{*}(B \mathrm{DI}(4) ; R)^{\otimes r} \otimes H^{*}\left(\mathbb{R} P^{\infty} ; R\right)^{\otimes s} \otimes H^{*}\left(\mathbb{C} P^{\infty} ; R\right)^{\otimes t}
$$


as a graded algebra, for some $r, s, t \geq 0$, where $G$ is a compact connected Lie group with finite center. In particular, if all generators of $P^{*}$ are in degree $\geq 3$ then $P^{*}$ is a tensor product of the cohomology rings of the classifying spaces of $\mathrm{SU}(n)$, $\operatorname{Sp}(n), \operatorname{Spin}(7), \operatorname{Spin}(8), \operatorname{Spin}(9), G_{2}, F_{4}$, and $\operatorname{DI}(4)$.

The proof reduces to $R=\mathbb{F}_{2}$, and then uses the classification of 2-compact groups. It would be interesting to try to list all polynomial rings which occur as $H^{*}\left(B G ; \mathbb{F}_{2}\right)$ for $G$ a compact connected Lie group with finite center.

One can also determine to which extend the space is unique. The following result was proved by Notbohm [81] for $p$ odd and 8 for $p=2$, as the culmination of a long series of partial results, started by Dwyer-Miller-Wilkerson [38, 39.

Theorem 4.3 (Uniqueness of spaces with polynomial $\mathbb{F}_{p}$-cohomology). Suppose $A^{*}$ is a finitely generated polynomial $\mathbb{F}_{p}$-algebra over the Steenrod algebra $\mathcal{A}_{p}$, with generators in degree $\geq 3$. Then there exists, up to $p$-completion, at most one homotopy type $Y$ with $H^{*}\left(Y ; \mathbb{F}_{p}\right) \cong A^{*}$, as graded algebras over the Steenrod algebra.

If $P^{*}$ is a finitely generated polynomial $\mathbb{F}_{p}$-algebra, then there exists at most finitely many homotopy types $Y$, up to $p$-completion, such that $H^{*}\left(Y ; \mathbb{F}_{p}\right) \cong P^{*}$ as graded $\mathbb{F}_{p}$-algebras.

The assumption $\geq 3$ above cannot be dropped, as easy examples show, and integrally uniqueness rarely hold, as discussed in Section 3 , see also 7,8 .

\section{Homotopical finite groups, group actions,...}

This survey is rapidly coming to an end, but we nevertheless want to briefly mention some other recent developments in homotopical group theory.

In connection with the determination of the algebraic K-theory of finite fields, Quillen and Friedlander proved the following: If $G$ is a reductive group scheme, and $q$ is a prime power, $p \nmid q$, then

$$
B G\left(\mathbb{F}_{q}\right)_{\hat{p}}^{\hat{p}} \simeq\left(B G(\mathbb{C})_{p}\right)^{h\left\langle\psi^{q}\right\rangle}
$$

where the superscript means taking homotopy fixed-points of the self-map $\psi^{q}$ corresponding to multiplication by $q$ on the root datum - it says that, at $p$, fixed-points and homotopy fixed-points of the Frobenius map raising to the $q$ th power agree.

The right-hand side of the equation makes sense with $B G(\mathbb{C})_{p}$ replaced by a $p$-compact group. Benson speculated in the mid 90s that the resulting object should be the classifying space of a " $p$-local finite group", and be determined by a conjugacy or fusion pattern on a finite $p$-group $S$, as axiomatized by Puig 83 . (motivated by block theory), together with a certain rigidifying 2-cocycle. He even gave a candidate fusion pattern corresponding to DI(4), namely a fusion pattern constructed by Solomon years earlier in connection with the classification of finite simple groups, but shown not to exist inside any finite group [12].

All this turns out to be true and more! A theory of $p$-local finite groups was founded and developed by Broto-Levi-Oliver in [20, and has seen rapid development by both homotopy theorists and group theorists since then. The Solomon 
2-local finite groups $\operatorname{Sol}(q)$ were shown to exist in [66, and a study of Chevalley $p$-local finite groups, $p$ odd, was initiated in 22. A number of exotic $p$-local finite groups have been found for $p$ odd, but the family $\operatorname{Sol}(q)$ remains the only known examples at $p=2$, prompting the speculation that perhaps they are the only exotic simple 2-local finite groups! Even partial results in this direction could have implications for the proof of the classification of finite simple groups. A modest starting point is the result in [18] that any so-called constrained fusion pattern comes from a (unique constrained) finite group - the result is purely group theoretic, and, while not terribly difficult, the only known proof uses techniques of a kind hitherto foreign to the classification of finite simple groups.

One can ask for a theory more general than $p$-local finite groups, broad enough to encompass both $p$-completions of arbitrary compact Lie groups and $p$-compact groups, and one such theory was indeed developed in 21, the so-called $p$-local compact groups. One would like to identify connected $p$-compact groups inside $p$ local compact groups in some group theoretic manner. This relates to the question of describing the relationship between the classical Lie theoretic structure and the $p$-fusion structure, mentioned several times before in this paper; the proof of the classification of $p$-compact groups may offer some hints on how to proceed.

In a related direction, one may attempt to relax the condition of compactness in $p$-compact groups to include more general types of groups; the paper [29] shows that replacing cohomologically finite by noetherian gives few new examples. An important class of groups to understand are Kac-Moody groups, and the paper [19] shows, amongst other things, that homomorphisms from finite $p$-groups to KacMoody groups still correspond to maps between classifying spaces. This gives hope that some of the homotopical theory of maximal tori, Weyl groups, etc. may also be brought to work in this setting, but the correct general definition of a homotopy Kac-Moody group is still elusive, the Lie theoretic definition being via generatorsand-relations rather than intrinsic. A good understanding of the restricted case of affine Kac-Moody groups and loop groups would already be very interesting.

Groups were historically born to act, a group action being a homomorphisms from $G$ to the group of homeomorphisms of a space $X$. In homotopy theory, one is however often only given $X$ up to an equivariant map which is a homotopy equivalence. Here the appropriate notion of an action is an element in the mapping space $\operatorname{map}(B G, B \operatorname{Aut}(X))$, where as before $\operatorname{Aut}(X)$ denotes the space of self-homotopy equivalences (itself an interesting group!). Homotopical group actions can also be studied one prime at a time, and assembled to global results afterwards. Of particular interest is the case where $X$ is a sphere. Spheres are non-equivariantly determined by their dimension, and self-maps by their degree. It turns out that something similar is true for homotopical group actions of finite groups on $p$-complete spheres [50]. But, one has to interpret dimension as meaning dimension function, assigning to each $p$-subgroup of $G$ the homological dimension of the corresponding homotopy fixed-point set, and correspondingly the degree is a degree function, viewed as an element in a certain $p$-adic Burnside ring. Furthermore there is hope to determine exactly which dimension functions are realizable. Understanding groups is homotopically open-ended... 


\section{References}

[1] J. F. Adams and C. W. Wilkerson. Finite $H$-spaces and algebras over the Steenrod algebra. Ann. of Math. (2), 111(1):95-143, 1980.

[2] J. Aguadé. Constructing modular classifying spaces. Israel J. Math., 66(1-3):23-40, 1989.

[3] K. K. S. Andersen. The normalizer splitting conjecture for $p$-compact groups. Fund. Math., 161(1-2):1-16, 1999.

[4] K. K. S. Andersen, T. Bauer, J. Grodal, and E. K. Pedersen. A finite loop space not rationally equivalent to a compact Lie group. Invent. Math., 157(1):1-10, 2004.

[5] K. K. S. Andersen and J. Grodal. The isogeny theorem for $p$-compact groups. In preparation.

[6] K. K. S. Andersen and J. Grodal. Automorphisms of $p$-compact groups and their root data. Geom. Topol., 12:1427-1460, 2008.

[7] K. K. S. Andersen and J. Grodal. The Steenrod problem of realizing polynomial cohomology rings. J. Topol., 1(4):747-760, 2008.

[8] K. K. S. Andersen and J. Grodal. The classification of 2-compact groups. J. Amer. Math. Soc., 22(2):387-436, 2009.

[9] K. K. S. Andersen, J. Grodal, J. M. Møller, and A. Viruel. The classification of p-compact groups for $p$ odd. Ann. of Math. (2), 167(1):95-210, 2008.

[10] T. Bauer, N. Kitchloo, D. Notbohm, and E. K. Pedersen. Finite loop spaces are manifolds. Acta Math., 192(1):5-31, 2004.

[11] D. J. Benson. Polynomial invariants of finite groups. Cambridge Univ. Press, 1993.

[12] D. J. Benson. Cohomology of sporadic groups, finite loop spaces, and the Dickson invariants. In Geometry and cohomology in group theory (Durham, 1994), LMS LNS 252, pages 10-23. Cambridge Univ. Press, 1998.

[13] A. Borel. Euvres: collected papers. Vol. II. Springer, 1983.

[14] R. Bott. An application of the Morse theory to the topology of Lie-groups. Bull. Soc. Math. France, 84:251-281, 1956.

[15] N. Bourbaki. Éléments de mathématique: Groupes et algèbres de Lie. Masson, Paris, 1982. Chapitre 9. Groupes de Lie réels compacts.

[16] A. K. Bousfield and D. M. Kan. Homotopy limits, completions and localizations. LNM 304. Springer, 1972.

[17] K. Bremke and G. Malle. Root systems and length functions. Geom. Dedicata, 72(1):83-97, 1998.

[18] C. Broto, N. Castellana, J. Grodal, R. Levi, and B. Oliver. Subgroup families controllling p-local finite groups. Proc. London Math. Soc. (3), 91(2):325-354, 2005.

[19] C. Broto and N. Kitchloo. Classifying spaces of Kac-Moody groups. Math. Z., 240(3):621-649, 2002.

[20] C. Broto, R. Levi, and B. Oliver. The homotopy theory of fusion systems. J. Amer. Math. Soc., 16(4):779-856, 2003.

[21] C. Broto, R. Levi, and B. Oliver. Discrete models for the $p$-local homotopy theory of compact Lie groups and p-compact groups. Geom. Topol., 11:315-427, 2007.

[22] C. Broto and J. M. Møller. Chevalley p-local finite groups. Algebr. Geom. Topol., 7:1809-1919, 2007.

[23] M. Broué. Reflection groups, braid groups, Hecke algebras, finite reductive groups. In Current Developments in Mathematics 2000, pages 1-107. Intl. Press, 2001. 
[24] W. Browder. Torsion in $H$-spaces. Ann. of Math. (2), 74:24-51, 1961.

[25] G. Carlsson. Equivariant stable homotopy and Segal's Burnside ring conjecture. Ann. of Math. (2), 120(2):189-224, 1984.

[26] G. Carlsson. Segal's Burnside ring conjecture and related problems in topology. In Proc. Intl. Congress of Mathematicians, Vol. 1 (Berkeley, 1986), pages 574-579, 1987.

[27] N. Castellana. Representacions homotopiques de grups p-compactes. $\mathrm{PhD}$ thesis, Universitat Autònoma de Barcelona, 1999.

[28] N. Castellana. On the $p$-compact groups corresponding to the $p$-adic reflection groups $G(q, r, n)$. Trans. Amer. Math. Soc., 358(7):2799-2819, 2006.

[29] N. Castellana, J. A. Crespo, and J. Scherer. Noetherian loop spaces. J. Eur. Math. Soc. (JEMS), to appear. arXiv:0903.1701

[30] A. Clark. On $\pi_{3}$ of finite dimensional $H$-spaces. Ann. of Math. (2), 78:193-196, 1963.

[31] A. Clark and J. Ewing. The realization of polynomial algebras as cohomology rings. Pacific J. Math., 50:425-434, 1974.

[32] C. R. Curjel and R. R. Douglas. On $H$-spaces of finite dimension. Topology, 10:385$389,1971$.

[33] M. Demazure. Exposé XXI. Donnees Radicielles. In Schémas en groupes. III (SGA 3), LNM 153, pages 85-155. Springer, 1962/1964.

[34] E. Dror, W. G. Dwyer, and D. M. Kan. An arithmetic square for virtually nilpotent spaces. Illinois J. Math., 21(2):242-254, 1977.

[35] W. Dwyer and A. Zabrodsky. Maps between classifying spaces. In Algebraic topology, Barcelona, 1986, pages 106-119. Springer, 1987.

[36] W. G. Dwyer. Lie groups and p-compact groups. In Proc. Intl. Congress of Mathematicians, Extra Vol. II (Berlin, 1998), pages 433-442, 1998.

[37] W. G. Dwyer and D. M. Kan. Centric maps and realization of diagrams in the homotopy category. Proc. Amer. Math. Soc., 114(2):575-584, 1992.

[38] W. G. Dwyer, H. R. Miller, and C. W. Wilkerson. The homotopic uniqueness of $B S^{3}$. In Algebraic topology, Barcelona, 1986, pages 90-105. Springer, 1987.

[39] W. G. Dwyer, H. R. Miller, and C. W. Wilkerson. Homotopical uniqueness of classifying spaces. Topology, 31(1):29-45, 1992.

[40] W. G. Dwyer and C. W. Wilkerson. A cohomology decomposition theorem. Topology, 31(2):433-443, 1992.

[41] W. G. Dwyer and C. W. Wilkerson. A new finite loop space at the prime two. J. Amer. Math. Soc., 6(1):37-64, 1993.

[42] W. G. Dwyer and C. W. Wilkerson. Homotopy fixed-point methods for Lie groups and finite loop spaces. Ann. of Math. (2), 139(2):395-442, 1994.

[43] W. G. Dwyer and C. W. Wilkerson. The center of a p-compact group. In The Čech centennial (Boston, 1993), pages 119-157. Amer. Math. Soc., 1995.

[44] W. G. Dwyer and C. W. Wilkerson. Product splittings for $p$-compact groups. Fund. Math., 147(3):279-300, 1995.

[45] W. G. Dwyer and C. W. Wilkerson. Normalizers of tori. Geom. Topol., 9:1337-1380, 2005 .

[46] E. M. Friedlander. Exceptional isogenies and the classifying spaces of simple Lie groups. Ann. Math. (2), 101:510-520, 1975. 
[47] M. Geck and G. Malle. Reflection groups. In Handbook of algebra. Vol. 4, pages 337-383. Elsevier, 2006.

[48] R. L. Griess, Jr. Elementary abelian p-subgroups of algebraic groups. Geom. Dedicata, 39(3):253-305, 1991.

[49] J. Grodal. Higher limits via subgroup complexes. Ann. of Math. (2), 155(2):405457, 2002.

[50] J. Grodal and J. H. Smith. Classification of homotopy G-actions on spheres. In preparation.

[51] P. Hilton and J. Roitberg. On principal $S^{3}$-bundles over spheres. Ann. of Math. (2), 90:91-107, 1969.

[52] H. Hopf. Über die Topologie der Gruppen-Mannigfaltigkeiten und ihre Verallgemeinerungen. Ann. of Math. (2), 42:22-52, 1941.

[53] J. E. Humphreys. Reflection groups and Coxeter groups. Cambridge Univ. Press, 1990.

[54] S. Jackowski and J. McClure. Homotopy decomposition of classifying spaces via elementary abelian subgroups. Topology, 31(1):113-132, 1992.

[55] S. Jackowski, J. McClure, and B. Oliver. Homotopy classification of self-maps of $B G$ via $G$-actions. I+II. Ann. of Math. (2), 135(2):183-226,227-270, 1992.

[56] S. Jackowski, J. McClure, and B. Oliver. Maps between classifying spaces revisited. In The Čech centennial (Boston, MA, 1993), volume 181 of Contemp. Math., pages 263-298. Amer. Math. Soc., 1995.

[57] S. Jackowski, J. McClure, and B. Oliver. Self-homotopy equivalences of classifying spaces of compact connected Lie groups. Fund. Math., 147(2):99-126, 1995.

[58] S. Jackowski and B. Oliver. Vector bundles over classifying spaces of compact Lie groups. Acta Math., 176(1):109-143, 1996.

[59] I. M. James. Bibliography on H-spaces. In H-Spaces (Neuchâtel, 1970), LNM 196, pages 137-156. Springer, 1971.

[60] A. Jeanneret and A. Osse. The $K$-theory of $p$-compact groups. Comment. Math. Helv., 72(4):556-581, 1997.

[61] V. G. Kac. Infinite-dimensional Lie algebras. Cambridge Univ. Press, 1990.

[62] R. M. Kane. The homology of Hopf spaces. North-Holland, 1988.

[63] J. Lannes. Sur les espaces fonctionnels dont la source est le classifiant d'un $p$-groupe abélien élémentaire. Inst. Hautes Études Sci. Publ. Math., (75):135-244, 1992.

[64] J. Lannes. Applications dont la source est un classifiant. In Proc. Intl. Congress of Mathematicians, Vol. 1 (Zürich, 1994), pages 566-573, 1995.

[65] J. Lannes. Théorie homotopique des groupes de Lie (d'après W. G. Dwyer et C. W. Wilkerson). Astérisque, (227):Exp. 776, 3, 21-45, 1995. Sém. Bourbaki, 1993/94.

[66] R. Levi and B. Oliver. Construction of 2-local finite groups of a type studied by Solomon and Benson. Geom. Topol., 6:917-990, 2002.

[67] J. P. Lin. Two torsion and the loop space conjecture. Ann. of Math. (2), 115(1):3591, 1982.

[68] G. Malle. Spetses. In Proc. Intl. Congress of Mathematicians, Vol. II (Berlin, 1998), pages 87-96, 1998.

[69] H. Miller. The Sullivan conjecture on maps from classifying spaces. Ann. of Math. (2), 120(1):39-87, 1984. 
[70] H. Miller. The Sullivan conjecture and homotopical representation theory. In Proc. Intl. Congress of Mathematicians, Vol. 1 (Berkeley, 1986), pages 580-589, 1987.

[71] S. A. Mitchell. Quillen's theorem on buildings and the loops on a symmetric space. Enseign. Math. (2), 34(1-2):123-166, 1988.

[72] J. M. Møller. Homotopy Lie groups. Bull. Amer. Math. Soc. (N.S.), 32(4):413-428, 1995.

[73] J. M. Møller. N-determined 2-compact groups. I. Fund. Math., 195(1):11-84, 2007.

[74] J. M. Møller. N-determined 2-compact groups. II. Fund. Math., 196(1):1-90, 2007.

[75] H. Nakajima. Invariants of finite groups generated by pseudoreflections in positive characteristic. Tsukuba J. Math., 3(1):109-122, 1979.

[76] G. Nebe. The root lattices of the complex reflection groups. J. Group Theory, 2(1):15-38, 1999.

[77] D. Notbohm. Homotopy uniqueness of classifying spaces of compact connected Lie groups at primes dividing the order of the Weyl group. Topology, 33(2):271-330, 1994.

[78] D. Notbohm. Classifying spaces of compact Lie groups and finite loop spaces. In Handbook of algebraic topology, pages 1049-1094. North-Holland, 1995.

[79] D. Notbohm. p-adic lattices of pseudo reflection groups. In Algebraic topology (Sant Feliu de Guíxols, 1994), pages 337-352. Birkhäuser, 1996.

[80] D. Notbohm. Topological realization of a family of pseudoreflection groups. Fund. Math., 155(1):1-31, 1998.

[81] D. Notbohm. Spaces with polynomial mod-p cohomology. Math. Proc. Cambridge Philos. Soc., 126(2):277-292, 1999.

[82] B. Oliver. Vector bundles over classifying spaces. In Proc. Intl. Congress of Mathematicians, Vol. II (Berlin, 1998), pages 483-492, 1998.

[83] Ll. Puig. Frobenius categories. J. Algebra, 303(1):309-357, 2006.

[84] D. Quillen. On the cohomology and $K$-theory of the general linear groups over a finite field. Ann. of Math. (2), 96:552-586, 1972.

[85] D. L. Rector. Loop structures on the homotopy type of $S^{3}$. In Symp. on Algebraic Topology (Seattle, 1971), LNM 249, pages 99-105. Springer, 1971.

[86] D. L. Rector. Subgroups of finite dimensional topological groups. J. Pure Appl. Algebra, 1(3):253-273, 1971.

[87] J.-P. Serre. Groupes d'homotopie et classes de groupes abéliens. Ann. of Math. (2), 58:258-294, 1953.

[88] J.-P. Serre. Sous-groupes finis des groupes de Lie. Astérisque, (266):Exp. 864, 5, 415-430, 2000. Sém. Bourbaki, 1998/99.

[89] G. C. Shephard and J. A. Todd. Finite unitary reflection groups. Canadian J. Math., 6:274-304, 1954.

[90] T. A. Springer. Linear algebraic groups. Birkhäuser, second edition, 1998.

[91] J. D. Stasheff. $H$-space problems. In $H$-spaces (Neuchâtel, 1970), LNM 196, pages 122-136. Springer, 1971.

[92] N. E. Steenrod. The cohomology algebra of a space. Enseignement Math. (2), 7:153-178 (1962), 1961.

[93] N. E. Steenrod. Polynomial algebras over the algebra of cohomology operations. In H-spaces (Neuchâtel, 1970), LNM 196, pages 85-99. Springer, 1971. 
[94] D. Sullivan. Genetics of homotopy theory and the Adams conjecture. Ann. of Math. (2), 100:1-79, 1974.

[95] D. Sullivan. Geometric topology: localization, periodicity and Galois symmetry, volume 8 of K-Monographs in Math. Springer, 2005. The 1970 MIT notes.

[96] J. Tits. Normalisateurs de tores. I. Groupes de Coxeter étendus. J. Algebra, 4:96116, 1966.

[97] T. tom Dieck. Geometric representation theory of compact Lie groups. In Proc. Intl. Congress of Mathematicians, Vol. 1 (Berkeley, 1986), pages 615-622, 1987.

[98] B. Totaro. Towards a Schubert calculus for complex reflection groups. Math. Proc. Cambridge Philos. Soc., 134(1):83-93, 2003.

[99] C. W. Wilkerson. Rational maximal tori. J. Pure Appl. Algebra, 4:261-272, 1974.

[100] C. W. Wilkerson. Applications of minimal simplicial groups. Topology, 15(2):111130, 1976.

[101] A. Zabrodsky. On the realization of invariant subgroups of $\pi_{*}(X)$. Trans. Amer. Math. Soc., 285(2):467-496, 1984.

[102] K. Ziemiański. A faithful unitary representation of the 2-compact group DI(4). J. Pure Appl. Algebra, 213(7):1239-1253, 2009.

Department of Mathematical Sciences, University of Copenhagen

Universitetsparken 5, DK-2100 Copenhagen, Denmark

E-mail: jg@math.ku.dk 